\title{
Our experience in sperm morphology assessment
}

\author{
Julia Irene Ariagno, Susana Mercedes Curi, Patricia Chenlo, Herberto Ernesto Hector Repetto, \\ Mercedes Norma Pugliese, Luis Alberto Palaoro, Melba Sardi and Gabriela Ruth Mendeluk
}

Asian Journal of Andrology (2011) 13, 201-202; doi:10.1038/aja.2010.119; published online 15 November 2010

We read with great interest the article recently published by Menkeveld et al. ${ }^{1}$ as we have a double interest in sperm morphology assessment. The first one as a Clinical Andrology Laboratory (Laboratory of Male Fertility, Department of Clinical Biochemistry, Faculty of Pharmacy and Biochemistry, University of Buenos Aires, Argentina) attempting to give valuable diagnosis to our patients, and the second one as Sponsors of the External Quality Control for the Study of Human Semen in Argentina (Programa de Evaluación Externa de la Calidad (PEEC) de la Fundación Bioquímica Argentina ${ }^{2}$ ). Beyond that we are particularly interested in giving our opinion and assessing the new edition of the WHO Laboratory Manual for the Examination and Processing of Human Semen. It is still perfectible with the expert contribution of staff working in the field. Our major aim is that finally it should include relevant guidelines for the correct assessment of semen all over the world.

Our purpose is to transmit our team's laboratory experience, where more than 55000 semen studies have been performed over the last 40 years. As mentioned in point 4 of Menkveld's article, we also wondered about the possible reasons for the decline in reference values. In 2008 we re-evaluated semen smears $(n=80)$, according to WHO guidelines. ${ }^{3}$ Figure 1 shows data on sperm morphology. According to our data, we consider that Menkeveld's first and second hypotheses are in accordance with ours as: (i) the implementation of strict sperm morphology evaluation has become overcritical; and (ii) over the years more criteria for sperm morphological

Laboratory of Male Fertility, Department of Clinical Biochemistry, INFIBIOC, Faculty of Pharmacy and Biochemistry, University of Buenos Aires, Buenos Aires 1120, Argentina

Correspondence: Dr J Ariagno (jiariagno@yahoo.com.ar) Received: 19 August 2010; Revised: 6September 2010; Accepted: 10 September 2010 abnormalities have been identified and introduced into the evaluation system. We could not demonstrate a true decline in sperm morphology in the period studied.

In the final paragraph of the item 2.3 titled: evolution of WHO criteria for sperm morphology, Dr Menkeveld affirms that the fourth (1999) edition of the WHO manual ${ }^{4}$ completely adopted the strict criteria for sperm morphology assessment. ${ }^{5}$ We agree with Dr Menkeveld's statement in item 6: 'the results of sperm morphology evaluations can be further improved by better international standardization for the whole sperm morphology evaluation procedure, better international training and improvement, and standardization of international external quality control (EQC) schemes'. We distribute to our EQC program participants, formalin-fixed semen samples for sperm count, videos for motility and a CD for morphology to avoid further errors to the standardization of the test owing to different staining techniques. These have been clearly illustrated by Cooper et al. ${ }^{6}$ and reinforced by Henkel et al. ${ }^{7}$
In a survey regarding pre-analytical and analytical aspects of sperm studies done by 77 participating laboratories (Argentinian EQC program), only $32 \%$ considered that WHO 1999 criteria and Kuger's are the same, $40 \%$ responded that they are different while $28 \%$ did not express their position on this subject. Discarding many other procedural concepts, we highlighted here that, at least in our country, there is still basic confusion on sperm morphology assessment, leading to the great interlaboratory differences detected by us. Also in the literature these criteria appear to be confused. ${ }^{8}$ Consensus on the interpretation of sperm morphology evaluation criteria should not be done if the criteria are not clearly known by the technicians. This is a subject of education, the first simple step to reach the larger goal.

In the tutorial located in the new manual, only one observer, Dr Kruger defined with subjective criteria which were morphologically normal spermatozoa and which were not. Is this statement acceptable today when there is an emerging consensus that at least a median from several experts is expected? ${ }^{9}$

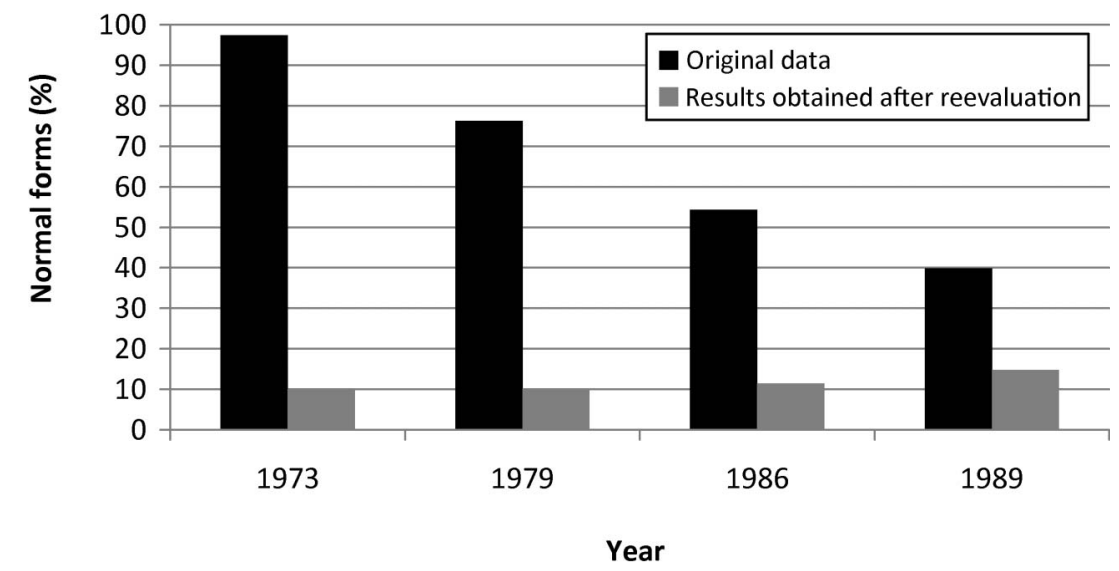

Figure 1 The mean value for the month of November according to the original data and post re-evaluation by the criterion WHO 1999. 
neg

Commentary

202

To investigate this point we designed a test in which we sent to 20 expert operators 150 micrograph of spermatozoa which appear in the new edition of the manual, but in a different order, some being repeated. There was $100 \%$ agreement on the assessment of the spermatozoa assessed as abnormal by Dr Kruger in the new manual. ${ }^{10}$ On the contrary, while analyzing normal spermatozoa such as those in plate 1 , pictures: $3,4,8,19$ and 20; or those with slight anomalies such as those in plate 4, pictures: 6, 7 and 11-15; results were frankly discrepant between the experts and even by the same observer on different occasions. This experience verifies the subjectivity of the test. As previously stated, data on sperm morphology show a high dispersion not only between laboratories, as intra-operator uncertaintanty was also revealed in our EQC program, proving that the major uncertainty lies in the classifiction of the borderline forms. Our sagestimon is not to consider the borderline forms as abnormal.

ACKNOWLEDGMENTS

This work is supported by a grant from University of Buenos Aires Science and Technology (UBCYT B031 Argentina).

1 Menkveld R. Clinical significance of the low normal sperm morphology value as proposed in the fifth edition of the WHO Laboratory Manual for the Examination and Processing of Human Semen. Asian J Androl 2010; 12: 47-58.

2 Cur S, Ariagno J, Chenlo P, Pugliese N, Sadi M et al. Control de Calidad en el Estudio del Semen. Act Bioquím Clín Latinoam 2008; 42: 183-7.

3 Ariagno JI, Chenlo PH, Sadi M, Pugliese NM, Mendeluk GR et al. Laboratorio de fertilidad masculine: Criterion de evaluación de la morfología espermática. Buenos Aires, Argentina: Faba Informa; 2009.
4 World Health Organization. WHO Laboratory Manual for the Examination of Human Semen and SpermCervical Mucus Interaction. 4th ed. New York: Cambridge University Press; 1999.

5 Menkveld R, Stander FS, Kotze TJ, Kruger TF, van Zyl $J A$. The evaluation of morphological characteristics of human spermatozoa according to stricter criteria. Hum Reprod 1990; 5: 586-92.

6 Cooper TG, Björndahl L, Vreeburg J, Nieschlag E. Semen analysis and external quality control schemes for semen analysis need global standardization. Int J Androl 2002; 25: 306-11.

7 Henkel R, Schreiber G, Sturmhoefel A, Hipler UC, Kerman $\mathrm{DH}$ et al. Comparison of three staining methods for the morphological evaluation of human spermatozoa. Fertil Steril 2008; 89: 449-55.

8 Nallella KP, Sharma RK, Aziz N, Agarwal A. Significance of sperm characteristics in the evaluation of male infertility. Fertil Steril. 2006, 58: 629-34.

9 Ricós C, Alvarez V, Hernández A, Jimenez CV, Minchinela $\mathrm{J}$ et al. Approaches for providing target values to improve usefulness of external quality assessment scheme. The Spanish experience. Scan J Olin Lab Invest Suppl 1993; 212: 19-27.

10 World Health Organization. Laboratory Manual for the Examination and Processing of Human Semen. Fifth ed. Geneva: WHO; 2010.

Asian Journal of Andrology 